\title{
Optical spectroscopy of crystal nucleation one nucleus at a time
}

Oscar Urquidi ${ }^{1, \dagger}$, Johanna Brazard ${ }^{1, \dagger}$, Natalie LeMessurier ${ }^{2}$, Lena Simine ${ }^{2}$, Takuji B. M. Adachi ${ }^{1, *}$

Affiliations:

${ }^{1}$ Department of Physical Chemistry, Sciences II, University of Geneva, 30, Quai Ernest Ansermet, 1211 Geneva, Switzerland

${ }^{2}$ Department of Chemistry, McGill University, 801 Sherbrooke Street W, Montreal, Quebec H3A 0B8, Canada

*Correspondence to: Takuji.Adachi@unige.ch

† These authors contributed equally to this work

\begin{abstract}
While crystallization is a ubiquitous and an important process, the microscopic picture of crystal nucleation is yet to be established in spite of decades of dedicated research. ${ }^{1,2}$ Recent studies agree that the nucleation process is more complex than the view offered by the classical nucleation theory. ${ }^{3-8}$ Although optical spectroscopy has the potential to capture the details of nucleation dynamics, its application has been hampered by the stochastic and heterogeneous nature of crystal nucleation. Here, we implement an innovative Single Nucleus Spectroscopy (SNS) by combining Raman microspectroscopy and optical trapping induced crystallization (OTIC) to investigate crystallization one nucleus at a time. Raman spectral evolution during a single glycine crystal nucleation measured by SNS and analyzed by a non-supervised spectral decomposition technique uncovered the Raman spectrum of pre-nucleation aggregates as well as its critical role as an intermediate species in the dynamics. The agreement between the Raman spectrum of pre-nucleation aggregates and our simulated spectrum from glycine solutions further suggested that loosely-bound linear chains of glycine are the likely precursors of crystallization. These results highlight the power of performing optical spectroscopy at one nucleus at a time and could change how optical spectroscopy tackles the crystal nucleation problem.
\end{abstract}


Crystallization is an important process in a wide range of disciplines from fundamental science to industrial application. ${ }^{1,9,10}$ Despite the importance of controlling the crystallization and its morphology (e.g. polymorphism), the lack of microscopic description of crystal nucleation often limits the rational approach to its engineering and control. ${ }^{9} \mathrm{~A}$ major challenge in establishing the molecular level understanding of crystal nucleation is its stochastic and heterogeneous nature at the nanoscale. ${ }^{1}$ This has long prevented experimentalists to directly observe the nucleation event in real-time. Statistical approaches such as counting nucleation rate under various crystallization condition have been a major experimental method to circumvent the difficulty. ${ }^{1,2}$ The discrepancy between the nucleation rate obtained from the statistical experiments and the one predicted by classical nucleation theory (CNT) has led to a general agreement that crystal nucleation is a more complex phenomenon than how it is described by $\mathrm{CNT}^{2-5}$

A real-time observation of nucleation dynamics has been long desired as a critical step in establishing its microscopic picture. For example, crystallization of colloidal particles has been used as a model system to mimic the dynamics of crystallization because the assembly process of particles can be easily visualized in real-time by optical microscopy. ${ }^{11,12}$ Recent breakthrough in tackling this problem was the development of cryo- and liquid cell transmission electron microscopy (TEM) for visualizing crystallization of atomic/molecular systems. ${ }^{13-15}$ The presence of morphologically "featureless" or "amorphous" clusters was confirmed in these TEM studies, ${ }^{7,16}$ and insitu TEM results caught the moments that the clusters became well-defined crystals. ${ }^{6,17,18}$ More and more studies show the involvement of pre-nucleation aggregates in crystal nucleation process and confirm the complexity of crystal nucleation process in contrast to CNT. A key question is to understand the structural dynamics of pre-nucleation aggregates toward the phase transition.

Optical spectroscopy could be a powerful technique to extract the molecular level details of the structural dynamics of pre-nucleation aggregates towards the phase transition. The application of optical spectroscopy on crystal nucleation problem has been, however, limited because the stochastic and heterogeneous nature of the nucleation process is detrimental to the interpretation of the spectroscopic signals. If one probes a large volume of a sample to capture a nucleation event that can occur anywhere 
at any time, the obtained spectroscopic signal is obscured by the average of various species in solution (e.g. monomers, aggregates, and crystals). Although not applied in this field yet, there is a wellestablished powerful concept to deal with a stochastic, complex and heterogenous system: Single molecule spectroscopy. ${ }^{19}$ The key to bring optical spectroscopy with its full potential is to probe single nucleation event at a time, only if we can predict precisely where a nucleation occurs.

Herein, we report the first demonstration of single nucleus spectroscopy (SNS) which spectroscopically probes crystal nucleation process one nucleus at a time. SNS is based on a novel extension of optical trapping Raman micro-spectroscopy, a well-established tool to study a trapped single object. ${ }^{20-22}$ In this work, optical trapping was used to induce a crystal nucleation from a focused laser spot instead of as a tweezer to trap an object. Optical trapping induced crystallization (OTIC) was first demonstrated by Sugiyama et al. in 2007 by focusing a near infrared (NIR) laser in supersaturated glycine/ $\mathrm{D}_{2} \mathrm{O}$ solution. ${ }^{23}$ Since the discovery, OTIC has been applied to a variety of systems, ${ }^{24-26}$ and furthermore high quality single crystals can be prepared while the polymorphs can be controlled by laser polarization. ${ }^{26,27}$ Our approach applies OTIC to spatially control a single crystal nucleation event (i.e. at a focused laser spot), so that a probe beam can be placed at the same position to track the nucleation process. We achieved measuring Raman spectral evolution of a single glycine crystal formation in solution with $46 \mathrm{~ms}$ time resolution at room temperature. The fast spectral acquisition allowed us to extract the Raman spectrum of pre-nucleation aggregates and its kinetics during a nucleation. The comparison between the experimental and simulated Raman spectra of glycine aggregates provided new insights into the glycine crystallization dynamics such as the formation of loosely-bound linear chains toward the nucleation.

\section{Results and Discussion}

The in-situ SNS setup to track single crystal nucleation dynamics was custom built based on an inverted microscope (Fig. 1a). While OTIC has been performed using a NIR laser in the past, ${ }^{23-28}$ we employed a $532 \mathrm{~nm}$ laser that served the dual role of inducing a crystal nucleation and Raman excitation (Fig. 1b). This design has two major benefits: i) the setup is as simple as conventional confocal microscope; ii) high laser power (> $1 \mathrm{~W}$ at a sample) required to induce crystallization produces high 
Raman signal (c.f. typical Raman spectroscopy is performed using $\sim 10 \mathrm{~mW}$ with long accumulation time), which increases the temporal resolution of Raman spectroscopy (46 ms time resolution in this report). The other benefits of a $532 \mathrm{~nm}$ laser compared with a NIR laser is that Raman scattering is more efficient and the temperature of water at laser focus does not increase $\left(\sim 2 \mathrm{mK} \mathrm{W}^{-1}\right) \cdot{ }^{29}$ The polarization of the laser beam was randomized by a depolarizer to minimize any laser polarization effect on crystallization process to mimic a "natural" crystallization occurring in solution. This also simplified the interpretation of Raman spectroscopy by eliminating Raman spectral variation due to polarization selection rules.
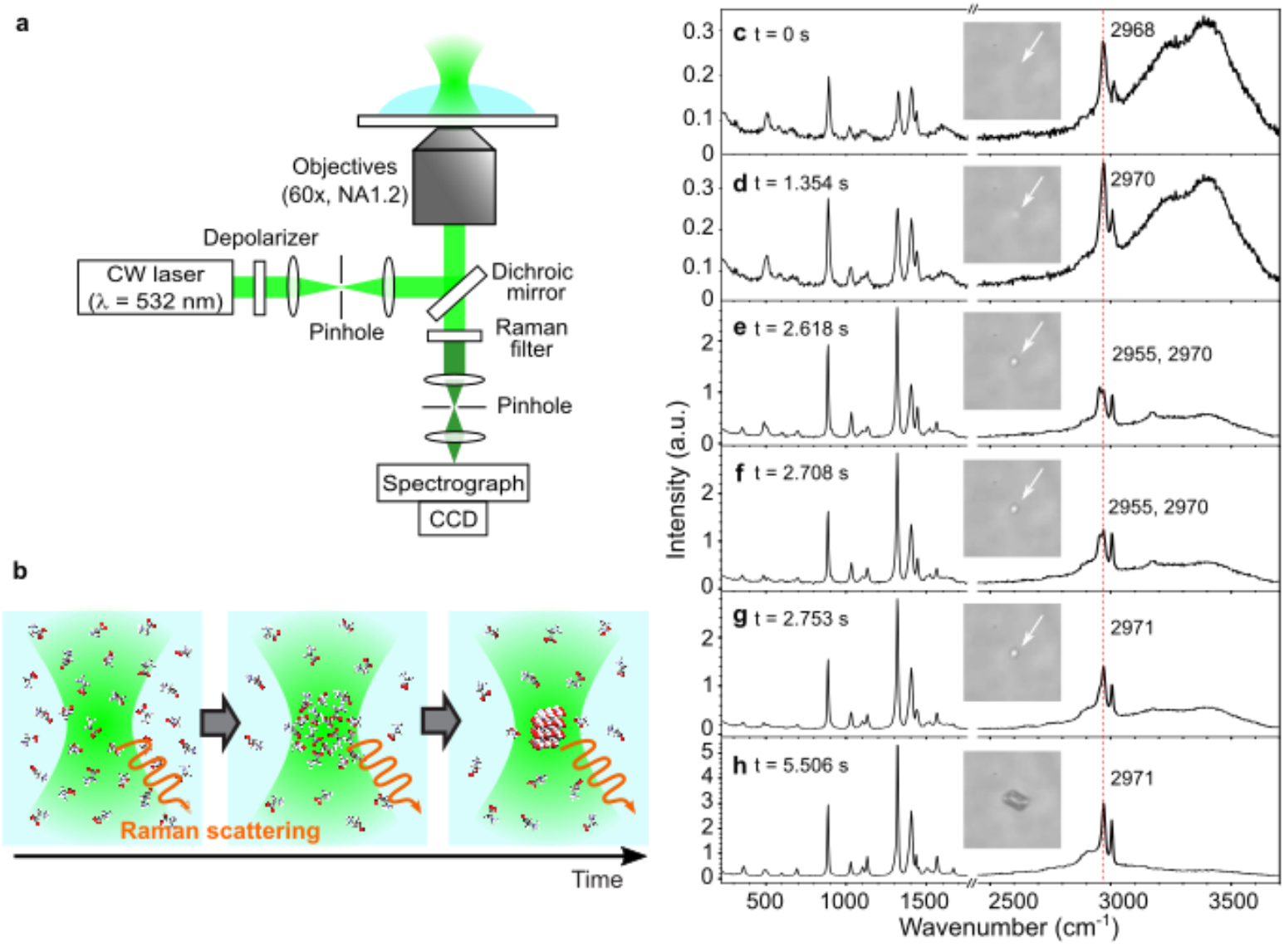

Fig. 1 Single Nucleus Spectroscopy (SNS): In-situ Raman spectroscopy during a-glycine crystallization at singe nucleus at a time. a, A simple scheme of the home-built SNS setup to achieve in-situ Raman measurement during single crystal nucleation. b, A cartoon representing the dual role of $532 \mathrm{~nm}$ CW laser. A tightly focused CW laser induces crystallization at the focus while it serves as Raman excitation laser to track the crystallization dynamics. c-h. Snapshots (46 ms time resolution) of Raman spectral evolution during a-glycine crystallization. Insets are the bright field microscopy images $(16 \times 16 \mu \mathrm{m})$ taken at the corresponding time to each acquired spectrum. 
Glycine was chosen as a model system in this study. While glycine has been widely studied as it is the simplest amino acid, its crystallization process has been shown rather complex and is under active debates. ${ }^{30-36}$ Fig. 1c-f shows a series of Raman spectra obtained in-situ during a single crystal nucleation of glycine which highlights the dynamic nature of crystal nucleation (see the movie SI1 for the whole experiment). Over the course of a nucleation, broad Raman bands around $3200-3500 \mathrm{~cm}^{-1}$ due to $\mathrm{O}-\mathrm{H}$ vibration of water molecules decreased. This coincides with the appearance of a small blurry object in the bright field image starting from the frame Fig. 1d. These observations can be interpreted that the local concentration of glycine molecules increased and pushed water molecules out of the confocal detection volume. The formation of a crystal is clearly captured with the increase of Raman intensity and the appearance of new peaks (Fig. 1e) even if the bright field image remains blurry. Among various spectral changes upon nucleation, the appearance of the Raman peak at $2955 \mathrm{~cm}^{-1}$ caught our immediate attention because $\alpha$-glycine (C-H stretching mode: $\sim 2970 \mathrm{~cm}^{-1}$ ) was expected to form in water. From the comparison of the feature with the reference spectra measured on the same setup from $\alpha$-, $\beta$ - and $\gamma$-glycine (Fig. SI1), the species was identified as $\beta$-glycine. The Raman spectral feature of $\beta$-glycine was short-lived and quickly became that of $\alpha$-glycine (Fig. 1f-g).

The spectral evolution during a glycine nucleation was analyzed using non-negative matrix factorization (NMF), a type of unsupervised principal component analysis used in machine learning analysis (Fig. 2). ${ }^{37} \mathrm{NMF}$ has been used to deconvolute spectra in the field of Raman spectroscopy, and a powerful aspect of this analysis algorithm is that a set of partial Raman spectrum (PRS) as well as the amplitude of each PRS during the spectral evolution can be obtained without any assumption concerning the spectral shapes. The number of PRS used for the deconvolution is the only predetermined parameter. We first applied NMF analysis with two PRS to the spectral series in which clear feature of $\beta$-glycine was observed for several seconds before converting to $\alpha$-glycine, to minimize the complexity of the crystallization dynamics (Fig. 2a). The analysis with two PRS did not reach good fitting results (Fig. SI3), which indicates that the glycine crystal nucleation does not occur through CNT mechanism where the whole spectral evolution should be reproduced by a linear combination of the spectrum of solution and crystal. Instead, good quality fit was achieved with three PRS (Fig. 2b-f). One 
of the PRS (PRS-2) matches well with the Raman spectrum of $\beta$-glycine. Interestingly, the spectra before the appearance of $\beta$-glycine were deconvoluted to two PRS (PRS-1 and 3).
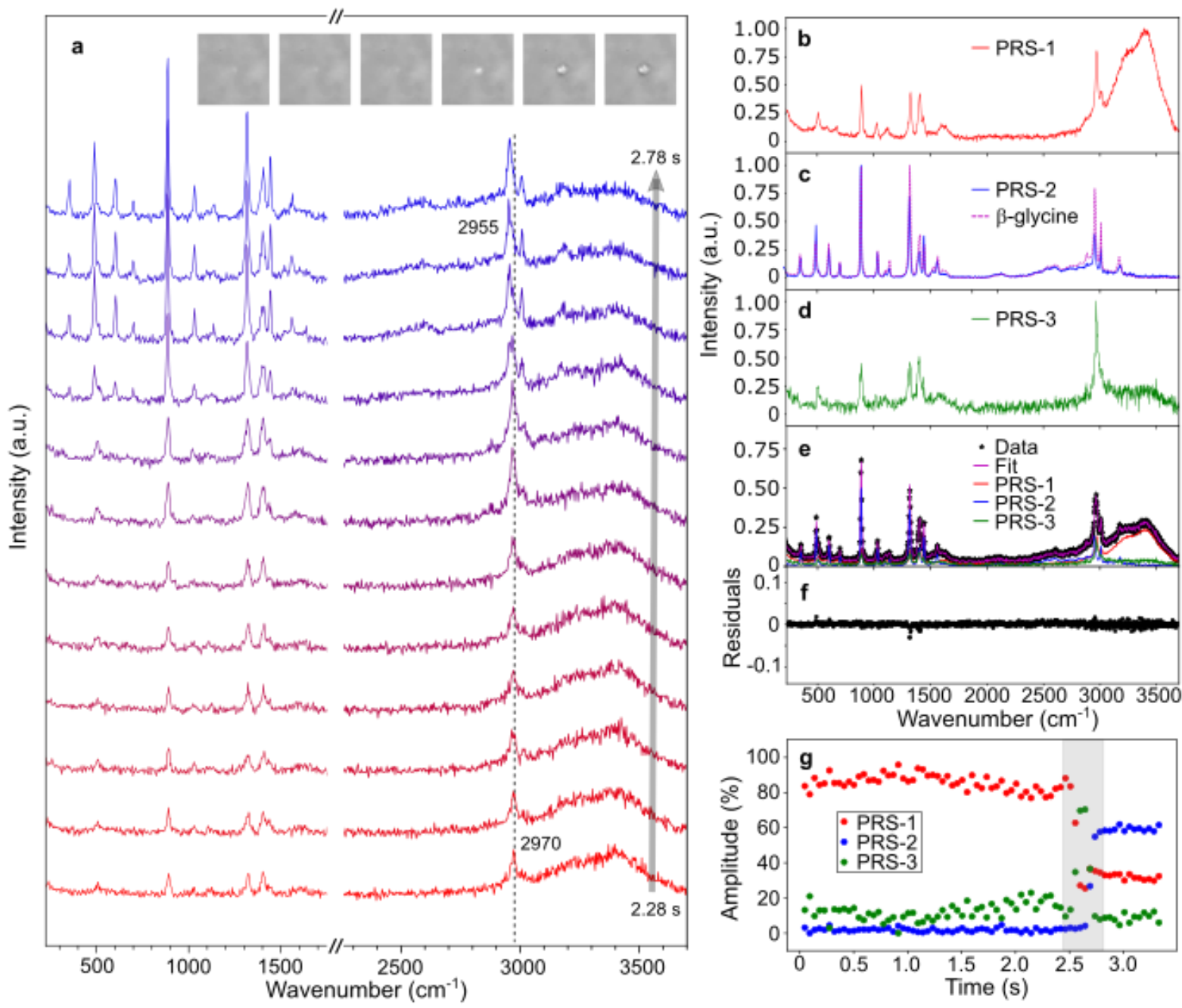

Fig. 2 Raman spectral evolution during a $\beta$-glycine crystal nucleation and its nonsupervised data decomposition analysis. a, Snapshots of Raman spectra showing the phase transition from solution (bottom) to crystal (top) with the bright field microscopy images (16x16 $\mu \mathrm{m})$ corresponding to every other spectrum (at $2.28 \mathrm{~s}$ to $2.78 \mathrm{~s}$ from the left to right) as insets. $\mathbf{b}-\mathbf{d}$, Three spectra obtained by non-supervised data decomposition of the series of spectra in the panel a (PRS: Partial Raman Spectrum 1-3). e, An example of the fit to the data (the frame at $2.692 \mathrm{~s}$ ) by three constituents and $\mathbf{f}$, the residuals of the fit. $\mathbf{g}$, Temporal evolution of each constituent amplitude during the crystal nucleation.

If we assumed that crystal nucleation occurred via non-classical nucleation pathway, PRS-1 can be interpreted as the Raman spectrum of monomers and PRS-3 as that of aggregates. This assumption fits well with the temporal evolution of each PRS amplitude (Fig. 2g). Initially, the spectra 
were composed of $80 \sim 85 \%$ of PRS-1, $0 \%$ of PRS-2 and 15 20\% of PRS-3. Just before the nucleation occurred at $\sim 2.65 \mathrm{~s}$ (the sudden increase of the amplitude of PRS-2), the amplitude of PRS-1 decreased while that of PRS-3 increased (highlighted by grey color). Once a crystal formed, the amplitude of PRS3 dropped down to almost zero. PRS-1 does not go down to zero at this time range, because there are remaining solution in the focal volume as the size of a crystal is still too small to occupy the whole focal volume. This kinetics was reproduced qualitatively in many experiments although there are some variances between the experiments due to the stochastic nature of crystallization (Fig. SI4 and Fig. SI5 for more examples).

It is worth noting that the amplitude of PRS-3 was steadily 15 20\% before the nucleation event. This could mean that the aggregates of glycine molecules are abandoned in a supersaturated aqueous solution. To test this hypothesis, we measured Raman spectrum of glycine aqueous solution as a function of concentration (Fig. 3a). To minimize an effect of optical trapping on the local concentration, a low laser power $(50 \mathrm{~mW})$ was used for this series of measurements. The intensity of Raman spectrum increased as the concentration, as expected. The intensity, however, did not increase proportionally as a function of the concentration but rather showed saturation behavior (Fig. SI6b). This suggests that the spectrum is composed of more than one species at higher concentration. In fact, NMF analysis with two PRS resulted in an excellent fit to the series of Raman spectra (Fig. 3b, PRS-1' and 2', Fig. SI7). The amplitude of PRS-1' is dominant at low concentration, and it decreases as the concentration increases while that of PRS-2' increases (Fig. 3c). From the concentration dependence on the amplitude, we assign PRS-1' as the spectrum of glycine monomers (dilute phase) and PRS-2' as that of glycine aggregates. 

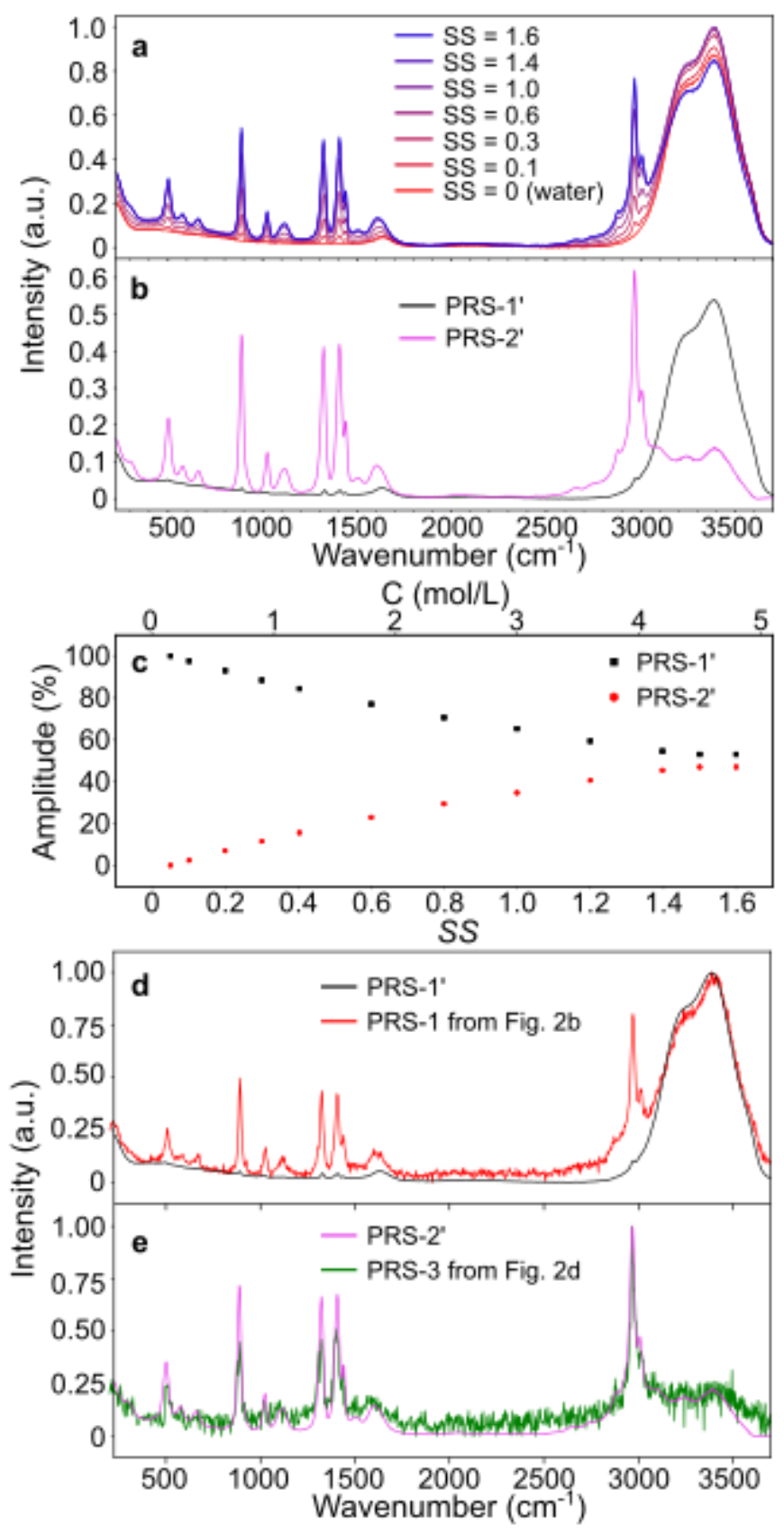

Fig. 3 A series of Raman spectra of glycine solution at different concentration and its NMF analysis revealing the spectrum of glycine monomers and aggregates. a, Raman spectra of glycine solution at different concentration. $\mathbf{b}$, Two PRS constituting the series of spectra in the panel a (PRS 1' and 2'), obtained from the NMF analysis. c, The amplitude of each component as a function of the concentration. $\mathbf{d}$, The overlay of PRS-1' and PRS-1 obtained from the nucleation dynamics (Fig. 2b), and e, PRS-2' and PRS-3 (Fig. 2d).

Remarkably, the PRS assigned as monomer (PRS-1') and aggregates (PRS-2') from the concentration series matches the spectral feature of PRS- 1 and 3 obtained from $\beta$-crystal nucleation dynamics (Fig. 3d-e). This strongly supports our interpretation of the observed nucleation dynamics (Fig.2) discussed earlier. Crystal nucleation of glycine occurs in non-classical pathway where pre- 
nucleation aggregates are formed and converted to a crystal. While there are always some aggregates present under the optical trapping laser, the rapid increase in the amplitude of aggregates which leads to crystal nucleation starts rather stochastically. It remains unclear what is the critical difference between the aggregates abandoned in solution and the pre-nucleation aggregates that convert to a crystal. As their Raman spectra matched well (Fig. 3e), aggregates that are present in solution and prenucleation aggregates may be structurally similar, except that we can only compare the time-averaged spectrum over $46 \mathrm{~ms}$. It will be crucial to track the structural fluctuation at faster time scale to gain further insight. The differences between individual aggregates and "pre-nucleation aggregates" will be certainly an important aspect to be spectroscopically explored in the future.

While the mechanism of glycine crystallization from aqueous solution has been under intense debates last decades, the direct comparison between simulated and experimental results has been challenging. This is the first time that Raman spectrum of pre-nucleation aggregates was identified, and this provides us a crucial link to the theoretical investigation of its structure. Recent studies based on molecular dynamics simulation suggested the presence of aggregates at high concentration of glycine aqueous solution, ${ }^{34,38,39}$ which agree with our observation. In this work, we were determined to gain an insight into the conformations of the pre-nucleation aggregates. To this end, we ran molecular dynamics simulations at two concentrations: low $\left(3.3 \mathrm{~mol} \mathrm{~L}^{-1}\right)$ representing the initial concentration in the experiments and high $\left(5.2 \mathrm{~mol} \mathrm{~L}^{-1}\right)$ modeling a putative concentration before nucleation. We have analyzed the sampled configurations (Fig. 4a-b) and performed Quantum Chemistry calculations to obtain Raman spectra from glycine clusters of varying sizes. The agreement between the simulated and the experimental Raman spectra (see Fig. 4c-e) has formed the basis for our interpretation of the computational results. 

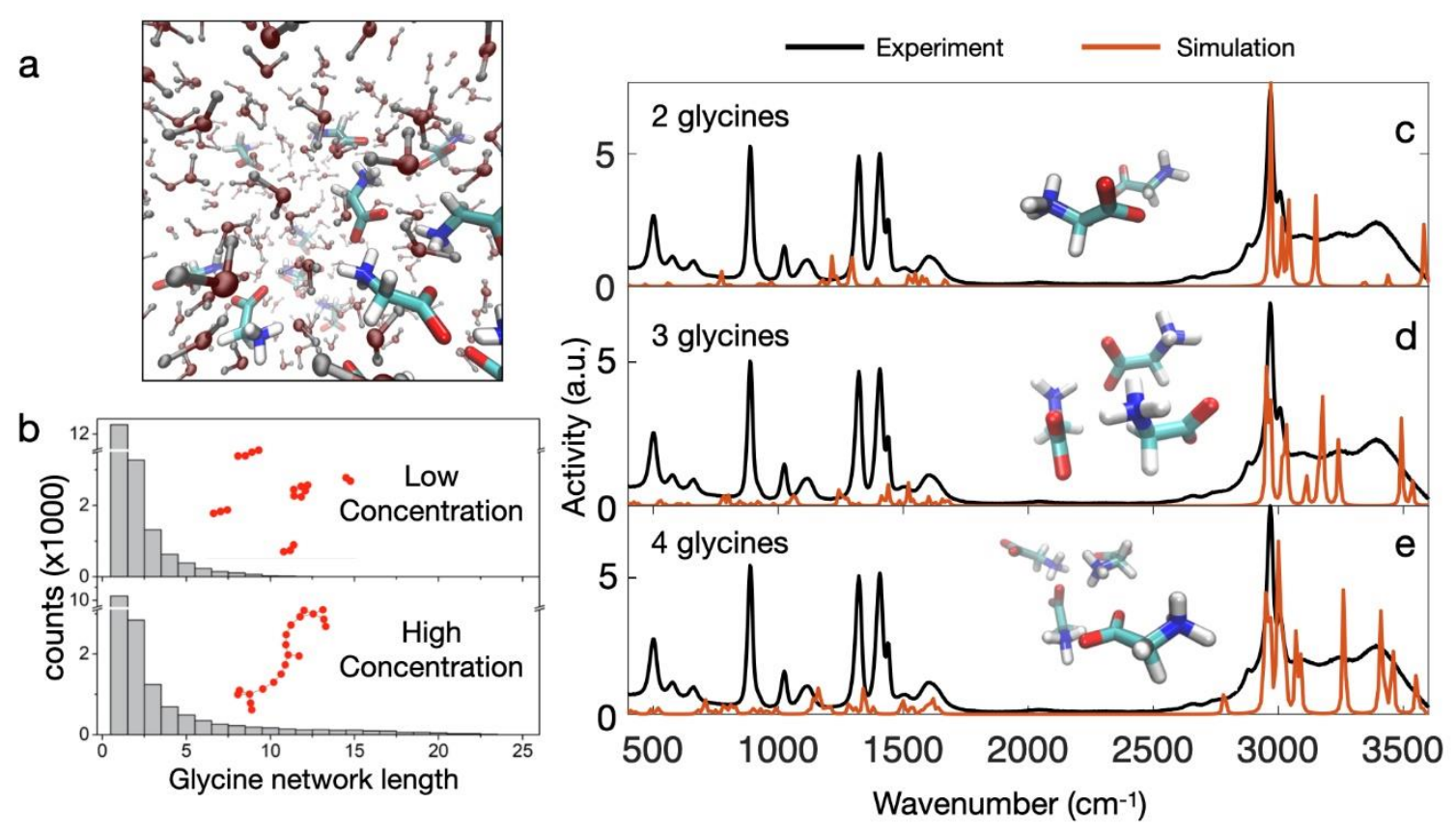

Fig. 4 Molecular dynamics (MD) simulation of glycine solutions and Quantum Chemistry calculation of the sampled glycine clusters. a, A snapshot from a MD trajectory of glycines in water; $\mathbf{b}$, the effect of concentration on the formation of glycine-glycine contacts: at low concentration glycine predominantly exist as monomers, and as the concentration increases loosely-bound linear chains form. The insets are showing samples from the network visualizations: red circles are individual glycines, and the lines between them indicate contacts. Disconnected glycines are omitted. c-e, (red) simulated Raman spectra from glycine clusters of increasing sizes sampled using MD: c) two, d) three, and e) four glycines; the experimental PRS2'assigned to aggregates is shown in black.

Snapshots obtained from the MD simulation of glycine aqueous solution (Fig.4a, Fig. SI8) showed that glycine forms loosely bound hydrogen-bonded linear chains within the simulation box. We call these networks "loose" in the sense that doubly hydrogen bonded cyclic dimers ${ }^{40}$ are almost never observed. Fig. $4 \mathrm{~b}$ shows the histograms of the lengths of glycine networks in our simulations. The histogram on the top shows that at low concentrations monomers and loose dimers dominate the solution. As the concentration increases larger clusters emerge. The insets show the results of the network visualization analysis: red circles are individual glycines and the lines between them indicate contacts (2.5 cut-off was used). The calculated Raman spectra for the clusters of two, three, four glycines (Fig. 4c-e) show that the peaks gradually 'fill in' the broad spectroscopic feature observed in experiments (black), especially in the region of $2500-3500 \mathrm{~cm}^{-1}$ as the size of the clusters increases. A broadening of Raman spectrum is resulting from the heterogeneity of the conformation of individual 
glycines in the hydrogen bonded clusters (See movie SI2 and SI3). The agreement between theory and experiment indicates that the structure of pre-nucleation aggregates may be loosely-bound linear chains.

Recent studies based on X-ray diffraction and solid-state NMR on flash cooled glycine aqueous solution proposed a new hypothesis that $\alpha$-glycine crystallization occurs via glycine dihydrates and $\beta$ glycine. ${ }^{33,35,41}$ The presence of glycine dihydrates in water at room temperature is yet to be confirmed. Our results suggest another nucleation pathway that loosely-bound linear chains of glycine clusters may be the precursors to constitute the hydrogen bonded networks observed in the crystal structure of $\beta$ glycine.

In summary, we demonstrated a novel in-situ optical spectroscopy to study crystallization dynamics of glycine one nucleus at a time. Spectral dynamics obtained during single glycine nucleation event from aqueous solution at room temperature along with non-supervised spectral decomposition analysis provided new evidences to understand the nucleation dynamics: i) glycine crystallization occurs via non-classical nucleation pathway where pre-nucleation aggregates grow and nucleate; ii) the comparison between the experiments and simulations using the Raman spectrum of pre-nucleation aggregates suggests that glycine molecules form loosely-bound linear chains which may be the precursor to $\beta$-glycine crystal. Experimental investigation of crystal nucleation by optical spectroscopy has been hampered by the stochastic and heterogenous nature of the nucleation. An in-situ spectroscopic approach presented in this study, "single nucleus spectroscopy", can be regarded as a basic platform where more advanced spectroscopy techniques can be coupled to. We believe this is a crucial breakthrough to bring more optical spectroscopy in to further accelerate the investigation of crystal nucleation.

\section{Methods (3000 words max)}

\section{Sample preparation}

Glycine (Sigma-Aldrich) was used without further purification. Glycine aqueous solution was prepared using a ultrapure water (MilliQ). Glycine was dissolved at $80^{\circ} \mathrm{C}$ using a ultrasonication, and 
filtered with a $0.2 \mu \mathrm{m}$ Target $2^{\mathrm{TM}}$ Nylon syringe filter (Thermo Scientific), and then slowly cooled down ( $\sim 3$ hours) to room temperature in a dry bath (Thermo Scientific). The degree of supersaturation (SS) was calculated using the solubility of glycine in water at $20{ }^{\circ} \mathrm{C}\left(0.225 \mathrm{~g} \mathrm{ml}^{-1}\right) .^{42}$

For SNS, a sample was assembled by placing a silicone isolator sheet $(0.25 \mathrm{~mm}$ thick, Grace Bio-Labs 664475) with a $10 \mathrm{~mm}$ hole on a piranha cleaned cover glass. The silicone sheet was cleaned in methanol before the use. $10 \mu \mathrm{L}$ of glycine aqueous solution ( $\mathrm{SS}=120 \%$ ) was dropped in the hole which resulted in a thin film of glycine solution (100 $\mu \mathrm{m}$ thickness). Another cover glass was placed on top to prevent the evaporation of water. No spontaneous crystallization was observed for $\sim 1$ hour.

Conventional crystallization was performed to obtain $\alpha-, \beta-$, and $\gamma$-glycine to measure Raman spectrum of each phase (Fig. SI1). $\alpha$-glycine was prepared by the slow evaporation of glycine aqueous solution. $\beta$-glycine was prepared by adding methanol to aqueous solution of glycine. $\gamma$-glycine was obtained by the slow evaporation of glycine aqueous solution with potassium nitrate as additives (see more details in Supplementary Information).

\section{Single Nucleus Spectroscopy (SNS) setup}

1) General scheme

The optical setup (Fig.1a) was home-built based on an inverted microscope (Olympus IX73). A $532 \mathrm{~nm}$ CW laser (Laser Quantum, Opus 532) was used with a dual role for optical trapping and Raman excitation. The diameter of the laser beam was properly adjusted by a telescope to slightly overfill the back aperture of water-immersion objective lens (Olympus UPLSAPO60XW, NA1.2). Liquid crystal polymer depolarizer (Thorlabs, DPP25-A) was used to randomize the polarization of the laser beam. A periodic retardation pattern of the depolarizer generates two focus spots due to diffraction. The beam after the depolarizer was once focused by a lens to spatially filter one of the spots and then was recollimated (Fig. SI9). A dichroic beamsplitter (AHF analysentechnik AG, Raman beamsplitter RT 532 rdc, F78-535) and a 532 nm RazorEdge ${ }^{\circledR}$ ultrasteep long-pass edge filter (Semrock, LP03-532RE25) were used to remove the excitation beam from Raman scattering. The signal was spatially filtered at the conjugate plane using a $25 \mu \mathrm{m}$ pinhole to remove the contribution of extrafocal volume. The size 
of the pinhole adopted in this setup is smaller than the one in typical setups $(\sim 100 \mu \mathrm{m})$. Therefore, the contribution of Raman signal from the extrafocal volume is significantly reduced and the spectral sensitivity to the nucleation dynamics occurring at the laser focus is improved. ${ }^{43}$ Raman spectrum was recorded by using a spectrograph (Andor, Kymera 193) and a CCD (Andor, iDus420) with a grating with a density of 900 1/mm blazed at $550 \mathrm{~nm}$ (Quantum Design AG, A-SR2-GRT-0900-0550).

2) Technical details of $i n$-situ glycine crystallization

The depolarized laser beam was focused just below $(\sim 1 \mu \mathrm{m})$ the liquid-air interface of glycine solution. While it has been reported that the height of the liquid-air interface continuously changes during OTIC with a $1064 \mathrm{~nm}$ laser, ${ }^{44}$ almost no change of the height was observed in the experiments with a $532 \mathrm{~nm}$ laser. This could simply be because there is no heating at the focus when $532 \mathrm{~nm}$ is used. OTIC with a $532 \mathrm{~nm}$ laser not only allows one to use aqueous solution instead of deuterated $\left(\mathrm{D}_{2} \mathrm{O}\right)$ solution, but also reduces complications related with heat-induced flows around the focus spot. Although the height of the liquid-air interface was stable, we also verified that the spectral shape near the liquid-air interface does not change due to the focus position relative to the interface (Fig. SI10). When the beam is focused at or above the interface, Raman signal is lower because the half or more of the focus probes air. Besides the intensity, the spectral shape remained unchanged.

\section{Data analysis}

The raw data were noise-filtered by Singular Value Decomposition (SVD, see Fig. SI2). The cleaned spectra were analyzed by Non-negative Matrix Factorization (NMF) using the Scikit_Learn library in Python..$^{45}$ The python code and the data are available on Yareta. For more details, see the Supplementary Information.

\section{Computational simulations}

Molecular dynamics simulations were run on the zwitterionic form of glycine in a $8 \mathrm{~nm}^{3}$ water box using the AMOEBA 2013 forcefield ${ }^{46}$ implemented in the OpenMM toolkit ${ }^{47}$ at two concentrations: $3.3 \mathrm{~mol} \mathrm{~L}-1$ and $5.2 \mathrm{~mol} \mathrm{~L}^{-1}$. The Raman spectra calculations were performed on clusters obtained 
from the MD trajectories using the Gaussian 16 suite of programs ${ }^{48}$ with B3LYP/6-311G(d) ${ }^{31}$. For the complete technical discussion, please see the Supplementary Information Section SI2.

\section{Acknowledgements}

T.B.M.A., O.U., and J.B. are thankful for the financial support from the University of Geneva. Computations were made on the supercomputer Béluga, managed by Calcul Québec (https://www.calculquebec.ca/) and Compute Canada (www.computecanada.ca). The operation of this supercomputer is funded by the Canada Foundation for Innovation (CFI).

\section{Author Contributions}

T.B.M.A. designed the initial experiments. T.B.M.A., O.U. and J.B. built the setup and finalized the design of the experiments. O.U. performed most of the measurements under supervision of T.B.M.A and J.B.. J.B. introduced NMF analysis, and O.U. and J.B. performed most of the NMF analysis. N.L and L.S. performed the computational simulations and analysis. All authors were involved in drafting the manuscript.

\section{Competing Interests Statement}

The authors declare no competing interests.

\section{References ( 30 max, can be 50 in special case)}

1. Mullin, J. W. Crystallization. (Elsevier Science, 2014).

2. Sosso, G. C. et al. Crystal Nucleation in Liquids: Open Questions and Future Challenges in Molecular Dynamics Simulations. Chemical Reviews 116, 7078-7116 (2016).

3. Erdemir, D., Lee, A. Y. \& Myerson, A. S. Nucleation of Crystals from Solution: Classical and Two-Step Models. Accounts of Chemical Research 42, 621-629 (2009). 
4. Gebauer, D., Kellermeier, M., Gale, J. D., Bergström, L. \& Cölfen, H. Pre-nucleation clusters as solute precursors in crystallisation. Chem. Soc. Rev. 43, 2348-2371 (2014).

5. Jehannin, M., Rao, A. \& Cölfen, H. New Horizons of Nonclassical Crystallization. J. Am. Chem. Soc. 141, 10120-10136 (2019).

6. Yamazaki, T. et al. Two types of amorphous protein particles facilitate crystal nucleation. Proc Natl Acad Sci USA 114, 2154-2159 (2017).

7. Tsarfati, Y. et al. Crystallization of Organic Molecules: Nonclassical Mechanism Revealed by Direct Imaging. ACS Central Science 4, 1031-1036 (2018).

8. Vekilov, P. G. The two-step mechanism of nucleation of crystals in solution. Nanoscale 2, 2346 (2010).

9. Bernstein, J. Polymorphism in molecular crystals. (Oxford University Press, 2002).

10. Tamura, R. \& Miyata, M. Advances in Organic Crystal Chemistry Comprehensive Reviews 2015. (Springer, 2015).

11. Schall, P., Cohen, I., Weitz, D. A. \& Spaepen, F. Visualizing dislocation nucleation by indenting colloidal crystals. Nature 440, 319-323 (2006).

12. Dong, F., Liu, M., Grebe, V., Ward, M. D. \& Weck, M. Assembly of Shape-Tunable Colloidal Dimers in a Dielectrophoretic Field. Chem. Mater. 32, 6898-6905 (2020).

13. De Yoreo, J. J. \& N. A. J. M., S. Investigating materials formation with liquid-phase and cryogenic TEM. Nat Rev Mater 1, 16035 (2016).

14. Patterson, J. P., Xu, Y., Moradi, M.-A., Sommerdijk, N. A. J. M. \& Friedrich, H. CryoTEM as an Advanced Analytical Tool for Materials Chemists. Acc. Chem. Res. 50, 1495-1501 (2017).

15. Ross, F. M. Opportunities and challenges in liquid cell electron microscopy. Science 350, aaa9886 (2015). 
16. Schreiber, R. E. et al. Real-time molecular scale observation of crystal formation. Nature Chemistry 9, 369-373 (2016).

17. Nielsen, M. H., Aloni, S. \& De Yoreo, J. J. In situ TEM imaging of CaCO3 nucleation reveals coexistence of direct and indirect pathways. Science 345, 1158-1162 (2014).

18. Nakamuro, T., Sakakibara, M., Nada, H., Harano, K. \& Nakamura, E. Capturing the Moment of Emergence of Crystal Nucleus from Disorder. J. Am. Chem. Soc. 143, 17631767 (2021).

19. Barbara, P. F. Single-Molecule Spectroscopy. Acc. Chem. Res. 38, 503-503 (2005).

20. Buajarern, J., Mitchem, L. \& Reid, J. P. Manipulation and Characterization of Aqueous Sodium Dodecyl Sulfate/Sodium Chloride Aerosol Particles. J. Phys. Chem. A 111, 13038-13045 (2007).

21. Chan, J. W. Recent advances in laser tweezers Raman spectroscopy (LTRS) for label-free analysis of single cells. J. Biophoton. 6, 36-48 (2013).

22. Mrad, R., Kruglik, S. G., Ben Brahim, N., Ben Chaâbane, R. \& Negrerie, M. Raman Tweezers Microspectroscopy of Functionalized 4.2 nm Diameter CdSe Nanocrystals in Water Reveals Changed Ligand Vibrational Modes by a Metal Cation. J. Phys. Chem. C 123, 24912-24918 (2019).

23. Sugiyama, T., Adachi, T. \& Masuhara, H. Crystallization of Glycine by Photon Pressure of a Focused CW Laser Beam. Chemistry Letters 36, 1480-1481 (2007).

24. Yuyama, K., Islam, M. J., Takahashi, K., Nakamura, T. \& Biju, V. Crystallization of Methylammonium Lead Halide Perovskites by Optical Trapping. Angew. Chem. Int. Ed. 57, 13424-13428 (2018).

25. Cheng, A.-C., Masuhara, H. \& Sugiyama, T. Evolving Crystal Morphology of Potassium Chloride Controlled by Optical Trapping. J. Phys. Chem. C 124, 6913-6921 (2020). 
26. Sugiyama, T., Yuyama, K. \& Masuhara, H. Laser Trapping Chemistry: From Polymer Assembly to Amino Acid Crystallization. Accounts of Chemical Research 45, 1946-1954 (2012).

27. Yuyama, K., Rungsimanon, T., Sugiyama, T. \& Masuhara, H. Selective Fabrication of $\alpha$ and $\gamma$-Polymorphs of Glycine by Intense Polarized Continuous Wave Laser Beams. Crystal Growth \& Design 12, 2427-2434 (2012).

28. Yuyama, K., Sugiyama, T. \& Masuhara, H. Laser Trapping and Crystallization Dynamics of L -Phenylalanine at Solution Surface. J. Phys. Chem. Lett. 4, 2436-2440 (2013).

29. Ito, S., Sugiyama, T., Toitani, N., Katayama, G. \& Miyasaka, H. Application of Fluorescence Correlation Spectroscopy to the Measurement of Local Temperature in Solutions under Optical Trapping Condition. J. Phys. Chem. B 111, 2365-2371 (2007).

30. Huang, J., Stringfellow, T. C. \& Yu, L. Glycine Exists Mainly as Monomers, Not Dimers, in Supersaturated Aqueous Solutions: Implications for Understanding Its Crystallization and Polymorphism. J. Am. Chem. Soc. 130, 13973-13980 (2008).

31. Friant-Michel, P. \& Ruiz-López, M. F. Glycine Dimers: Structure, Stability, and Medium Effects. Chem. Eur. J. of Chem. Phys. 11, 3499-3504 (2010).

32. Yani, Y., Chow, P. S. \& Tan, R. B. H. Glycine Open Dimers in Solution: New Insights into $\alpha$-Glycine Nucleation and Growth. Crystal Growth \& Design 12, 4771-4778 (2012).

33. Xu, W., Zhu, Q. \& Hu, C. T. The Structure of Glycine Dihydrate: Implications for the Crystallization of Glycine from Solution and Its Structure in Outer Space. Angew. Chem. 129, 2062-2066 (2017).

34. Bushuev, Y. G., Davletbaeva, S. V. \& Koifman, O. I. Molecular dynamics simulations of aqueous glycine solutions. CrystEngComm 19, 7197-7206 (2017). 
35. Cerreia Vioglio, P. et al. Insights into the Crystallization and Structural Evolution of Glycine Dihydrate by In Situ Solid-State NMR Spectroscopy. Angew. Chem. Int. Ed. 57, 6619-6623 (2018).

36. Broadhurst, E. T. et al. Polymorph evolution during crystal growth studied by 3D electron diffraction. IUCrJ 7, 5-9 (2020).

37. Woelffel, W. et al. Analysis of soda-lime glasses using non-negative matrix factor deconvolution of Raman spectra. Journal of Non-Crystalline Solids 428, 121-131 (2015).

38. Hamad, S., Hughes, C. E., Catlow, C. R. A. \& Harris, K. D. M. Clustering of Glycine Molecules in Aqueous Solution Studied by Molecular Dynamics Simulation. J. Phys. Chem. B 112, 7280-7288 (2008).

39. Di Gioacchino, M., Ricci, M. A., Imberti, S., Holzmann, N. \& Bruni, F. Hydration and aggregation of a simple amino acid: The case of glycine. Journal of Molecular Liquids 301, 112407 (2020).

40. Weissbuch, I., Torbeev, V. Yu., Leiserowitz, L. \& Lahav, M. Solvent Effect on Crystal Polymorphism: Why Addition of Methanol or Ethanol to Aqueous Solutions Induces the Precipitation of the Least Stable $\beta$ Form of Glycine. Angew. Chem. Int. Ed. 44, 3226$3229(2005)$.

41. Surovtsev, N. V. et al. Glycine phases formed from frozen aqueous solutions: Revisited. The Journal of Chemical Physics 137, 065103 (2012).

42. Datta, A. \& Hossain, A. Solubility Data of Glycine in Water and Justification of Literature Results. Asian J. Chem. 32, 1525-1533 (2020).

43. Wilson, T. Resolution and optical sectioning in the confocal microscope: PROPERTIES OF THE FLUORESCENT CONFOCAL MICROSCOPE. Journal of Microscopy 244, 113-121 (2011). 
44. Yuyama, K., Sugiyama, T. \& Masuhara, H. Millimeter-Scale Dense Liquid Droplet Formation and Crystallization in Glycine Solution Induced by Photon Pressure. The Journal of Physical Chemistry Letters 1, 1321-1325 (2010).

45. Pedregosa, F. et al. Scikit-learn: Machine Learning in Python. Journal of Machine Learning Research 12, 2825-2830 (2011).

46. Esser, A., Belsare, S., Marx, D. \& Head-Gordon, T. Mode specific THz spectra of solvated amino acids using the AMOEBA polarizable force field. Phys. Chem. Chem. Phys. 19, 5579-5590 (2017).

47. Eastman, P. et al. OpenMM 7: Rapid development of high performance algorithms for molecular dynamics. PLoS Comput Biol 13, e1005659 (2017).

48. Frisch, M. J. et al. Gaussian 16 Rev. C.01. (2016). 